\title{
Crystal structure of kindlin-2 PH domain reveals a conformational transition for its membrane anchoring and regulation of integrin activation
}

\author{
Yan Liu*, Yun Zhu*, Sheng Ye ${ }^{\varpi}$, Rongguang Zhang \\ National Laboratory of Biophysics, Institute of Biophysics, Chinese Academy of Sciences, Beijing 100101, China \\ $\bowtie$ Correspondence: rzhang@ibp.ac.cn (R. Zhang), yesheng@moon.ibp.ac.cn (S. Ye) \\ Received May 14, 2012 Accepted May 24, 2012
}

\begin{abstract}
Kindlin-2 belongs to a subfamily of FERM domain containing proteins, which plays key roles in activating integrin transmembrane receptors and mediating cell adhesion. Compared to conventional FERM domains, kindlin-2 FERM contains an inserted pleckstrin homology (PH) domain that specifically binds to phosphatidylinositol $(3,4,5)$ trisphosphate (PIP3) and regulates the kindlin-2 function. We have determined the crystal structure of kindlin-2 PH domain at $1.9 \AA$ resolution, which reveals a conserved $\mathrm{PH}$ domain fold with a highly charged and open binding pocket for PIP3 head group. Structural comparison with a previously reported solution structure of kindlin-2 PH domain bound to PIP3 head group reveals that upon PIP3 insertion, there is a significant conformational change of both the highly positively charged loop at the entry of the PIP3 binding pocket and the entire $\beta$ barrel of the $\mathrm{PH}$ domain. We propose that such "induced-fit" type change is crucial for the tight binding of PIP3 to anchor kindlin-2 onto the membrane surface, thereby promoting its binding to integrins. Our results provide important structural insight into kindlin-2mediated membrane anchoring and integrin activation.
\end{abstract}

KEYWORDS kindlin-2, integrin, $\mathrm{PH}$ domain, crystal structure, cell adhesion, membrane

\section{INTRODUCTION}

Integrins are major transmembrane receptors for cell adhe- sion, and also play important roles in mediating bidirectional signaling across the cell membrane for regulating cell migration, cell shape change, and survival (Hynes, 2002; Qin et al., 2004; Luo et al., 2007). They control diverse physiological processes such as immune response, leukocyte traffic, embryonic development, and homeostasis (Zhang and Wang, 2012). Each integrin contains a single $\alpha$ and $\beta$ subunit, respectively, which forms a heterodimer that undergoes conformational changes to transform the receptor from low-affinity state to high-affinity state (Vinogradova et al., 2002; Kim et al., 2003). Such conformational changes can be triggered by an "inside-out" signaling process that involves a series of intracellular proteins, notably talin and kindlin that bind to the integrin $\beta$ cytoplasmic tail (Moser et al., 2009). Talin is a cytoskeletal protein containing $\mathrm{N}$-terminal FERM (protein4.1, ezrin, radixin and moesin) domain and C-terminal rod domain. It has been well established that talin FERM domain binds to the integrin $\beta$ cytoplasmic tail and consequently activates integrins (Vinogradova et al., 2002; García-Alvarez et al., 2003; Anthis et al., 2009). Compared to talin, kindlin is a newer member of integrin activators (Moser et al., 2009; Plow et al., 2009). Increasing evidences have shown that kindlins bind to the distinct region of the integrin $\beta$ cytoplasmic tail and promote the integrin activation process in cooperation with talin (Shi et al., 2007; Moser et al., 2008; Harburger et al., 2009). Although there are different hypotheses about how kindlins mediate this activation process, its precise mechanism remains unclear.

Kindlin is named after the Kindler syndrome which is a skin blistering disease caused by kindlin gene mutation (Siegel et al., 2003). Near the C-terminus of kindlin, there is a FERM

\footnotetext{
*These authors contributed equally to the work.
} 


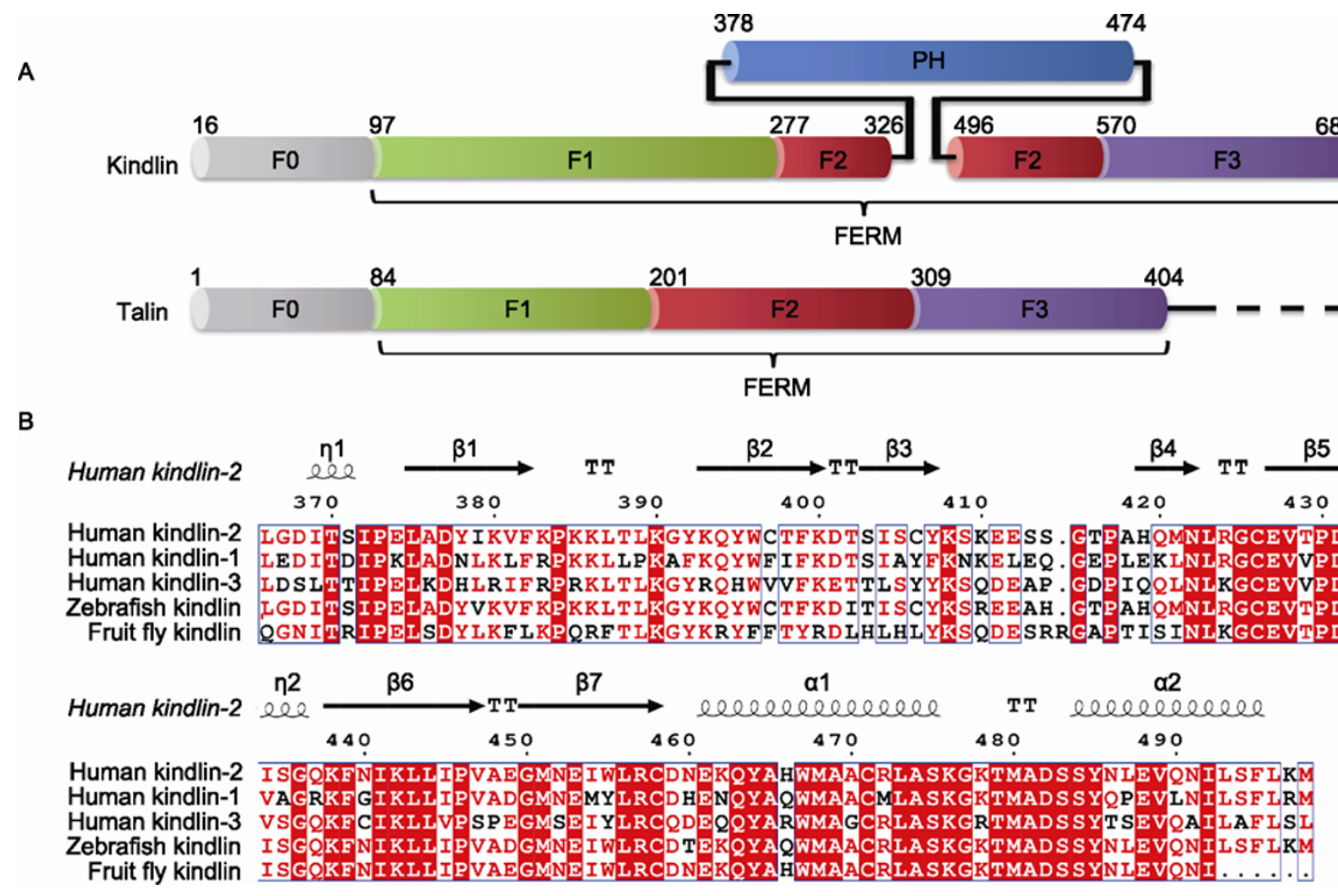

Figure 1. Kindlin-2 FERM domain contains an inserted conserved PH domain. (A) Diagram of the domain structure of kindlin-2 (top) and talin head domain (bottom), shown with residue numbers labeled. (B) Multiple sequence alignment of the kindlin $\mathrm{PH}$ domains from different isoforms and species. From top to bottom, the sequences are Homo sapiens kindlin-2, Homo sapiens kindlin-1, Homo sapiens kindlin-3, Danio rerio kindlin, and Drosophila melanogaster kindlin, respectively. Secondary structure elements of K2-PH are shown and labeled above the sequences.

domain similar to the FERM domain of talin. The major distinction between them is that an inserted pleckstrin homology $(\mathrm{PH})$ domain interrupts the F2 subdomain of kindlin FERM domain (Fig. 1A) (Moser et al., 2009; Plow et al., 2009). Since typical $\mathrm{PH}$ domain interacts with the negatively charged head group of specific membrane lipids (Lemmon, 2008), such insertion may promote the membrane anchoring of the kindlins and, thereby, regulate the kindlin-integrin interaction.

In human, the kindlin family includes three members, kindlin-1 (Unc-112-related protein 1, UPR1), kindlin-2 (Mig2), and kindlin-3 (URP-2). Among them, kindlin-2 was the first to be found to regulate integrin activation (Shi et al., 2007) and it does so by cooperating with talin (Ma et al., 2008; Montanez et al., 2008) and binding to membrane (Qu et al., 2011). Loss of kindlin-2 results in lethal phenotype in both $C$. elegans and mice (Rogalski et al., 2000; Montanez et al., 2008). Recent NMR studies of kindlin family members have characterized the structures of several isolated kindlin domains, including the $\mathrm{N}$-terminal ubiquitin-like domain of kindlin-2 (Perera et al., 2011), the $\mathrm{PH}$ domain of kindlin-2 (hereafter referred to as $\mathrm{K} 2-\mathrm{PH}$ ) in complex with IP4 (inositol 1,3,4,5-tetraphosphate, IP4) (Liu et al., 2011), and the PH domain of kindlin-3 (here- after referred to as K3-PH) (PDB entry: 2YS3). In particular, the complex structure containing IP4, the head group of membrane lipid phosphatidylinositol 3,4,5-triphosphate (PIP3), confirmed that the K2-PH is employed to fulfill the binding of kindlin-2 to the cytoplasmic surface of cell membrane (Liu et al., 2011). An attempt to crystallize K2-PH was reported to be successful (Lee et al., 2011), but no further crystal structure was deposited into Protein Data Bank (PDB) or reported.

In this study, we have determined the crystal structure of $\mathrm{K} 2-\mathrm{PH}$ in free form, representing the first crystal structure of a kindlin domain. Despite limited sequence homology, the kindlin-2 PH domain has a conserved fold as compared to other $\mathrm{PH}$ domains. Interestingly, comparison with the previous solution structure of $\mathrm{K} 2-\mathrm{PH}$ bound to IP4 unveils a significant difference in both the $\mathrm{PH} \beta$ barrel and the IP4 binding site, suggesting a conformational transition process upon the lipid binding. The result provides a dynamic view of the $\mathrm{K} 2-\mathrm{PH}$-membrane interaction process which is a vital step towards the kindlin-2-mediated integrin activation. The structure also sheds light upon the folding and function of the $\mathrm{PH}$ domains of other kindlin family members. 


\section{RESULTS AND DISCUSSION}

\section{A highly conserved PH domain in kindlin family}

Figure $1 \mathrm{~A}$ provides the comparison of kindlin-2 FERM domain and talin head, which clearly shows that there is an additional $\mathrm{PH}$ domain inserted in the F2 subdomain of kindlin-2. Typical $\mathrm{PH}$ domain structures include seven antiparallel $\beta$ strands and one amphiphilic $\alpha$ helix (two amphiphilic $\alpha$ helices in kindlin $\mathrm{PH}$ domain). Multiple sequence alignment of kindlin family from human, zebrafish, and fruit fly suggested that this $\mathrm{PH}$ domain is evolutionally conserved, especially in the $\beta 1, \beta 4, \beta 5, \beta 6$, and $\beta 7$ strands and $\alpha 1, \alpha 2$ helices (Fig.1B).

\section{The structure determination and overall architecture of K2-PH}

Multiple constructs including $\mathrm{K} 2-\mathrm{PH}$ and longer than the exact $\mathrm{K} 2-\mathrm{PH}$ domain were built for crystallographic studies. The one crystallized is from Ser328 to GIn499. Preliminary analysis of the $1.9 \AA$ diffraction data of K2-PH crystals indicated alternative possibilities of space groups $P 4_{1} 2_{1} 2$ and $P 4_{3} 2{ }_{1} 2$. Subsequent molecular replacement calculations determined that the real space group is $\mathrm{P}_{4} 2_{1} 2$. Both the solution structure of K2-PH bound to IP4 (PDB entry: 2LKO) and free form $\mathrm{K} 3-\mathrm{PH}$ (PDB entry: 2YS3) were employed as the starting model for molecular replacement. Intriguingly, it was the solution structure of free $\mathrm{K} 3-\mathrm{PH}$ that led to a reasonable phase solution, instead of that of K2-PH bound to IP4. A potential reason could be the significant difference between the structures of the free form $\mathrm{K} 2-\mathrm{PH}$ in our crystals and the IP4-complexed K2-PH in solution, which will be further discussed below.

In the final model of $\mathrm{K} 2-\mathrm{PH}$, there is one protein molecule in one asymmetric unit (AU), along with 87 water molecules and 2 tartaric acid groups from the precipitant for crystallization. Residues from Ile365 to GIn499 of K2-PH can be readily traced and refined according to the continuous electron-density map, while the first 47 residues at $\mathrm{N}$-terminus were hardly traceable and thus are missing in the final model. The crystallographic $R$-factor $\left(R_{\text {cryst }}\right)$ and the free $R$-factor $\left(R_{\text {free }}\right)$ of our final model is 0.195 and 0.224 , respectively. Further details and statistics are listed in Table 1.

As expected, $\mathrm{K} 2-\mathrm{PH}$ adopts a typical $\mathrm{PH}$ domain fold (Fig. 2A), which is generally similar to the previously reported solution structure (Fig. 2B). It is also similar to typical $\mathrm{PH}$ domains despite limited sequence homology (usually less than $20 \%$ identity) as represented by other $\mathrm{PH}$ domains, such as the $\mathrm{PH}$ domain of protein kinase B (Milburn et al., 2003) (hereafter referred to as PKB-PH) (Fig. 2C). At the very $\mathrm{N}$-terminus, there is a one-turn $3_{10}$ helix (green in Fig. 2A) consisting of residues Ile369 to lle372. Given the missing 47 $\mathrm{N}$-terminal residues and the flexible $\mathrm{N}$-terminus before $\beta 1$ in
Table 1 K2-PH diffraction data and refinement statistics

\begin{tabular}{|c|c|}
\hline \multicolumn{2}{|l|}{ Crystallographic data } \\
\hline $\mathrm{d}_{\min }(\AA)$ & $1.90(1.94-1.90)$ \\
\hline Wavelength $(\AA)$ & 1.5418 \\
\hline Measured reflections & 123,856 \\
\hline Average redundancy & $9.4(6.0)$ \\
\hline Mean $I / \sigma(I)$ & $7.0(1.9)$ \\
\hline Completeness (\%) & $99.9(100.0)$ \\
\hline$R_{\text {merge }}{ }^{a}$ & $0.063(0.688)$ \\
\hline \multicolumn{2}{|l|}{ Refinement statistics } \\
\hline Bragg spacing $(\AA)$ & $29.24-1.90$ \\
\hline Space group & $\mathrm{P} 4{ }_{3} 2{ }_{1} 2$ \\
\hline \multicolumn{2}{|l|}{ Cell parameters } \\
\hline$a, b(\AA)$ & 58.5 \\
\hline$c(\AA)$ & 92.2 \\
\hline Reflections in working set & 12,543 \\
\hline Reflections in test set & 652 \\
\hline$R_{\text {cryst }}{ }^{\mathrm{b}}$ & 0.195 \\
\hline$R_{\text {free }}{ }^{\mathrm{c}}$ & 0.224 \\
\hline r.m.s.d. bonds $(\AA)$ & 0.013 \\
\hline r.m.s.d. angles $\left({ }^{\circ}\right)$ & 1.5 \\
\hline Average $B$-factor $\left(\AA^{2}\right)$ & 34.2 \\
\hline Missing residues & 47 \\
\hline No. of waters & 86 \\
\hline
\end{tabular}

Values in parentheses indicate the corresponding statistics in the highest resolution shell.

${ }^{a} R_{\text {merge }}=\left(\Sigma \mid I_{\mathrm{i}}-\left\langle l_{\mathrm{i}}\right\rangle\right) / \Sigma\left|I_{\mathrm{i}}\right|$, where $l_{\mathrm{i}}$ is the integrated intensity of a given reflection.

${ }^{b} R_{\text {cryst }}=\left(\Sigma|| F_{\mathrm{o}}|-| F_{\mathrm{c}}||\right) / \Sigma\left|F_{\mathrm{o}}\right|$, where $F_{\mathrm{o}}$ and $F_{\mathrm{c}}$ denote observed and calculated structure factors, respectively.

${ }^{c} R_{\text {free }}$ is equivalent to $R_{\text {cryst }}$ but calculated using randomly chosen $5 \%$ reflections as the test set, which were excluded from refinement process.

our crystal structure, the precise topology of how the $\mathrm{PH}$ domain packs with the F2 subdomain in kindlins remains foggy. Residues Leu375 to Cys458 fold into a four-strand anti-parallel $\beta$ sheet (cyan in Fig. 2A), a three-strand one (blue in Fig. 2A), and a one-turn $3_{10}$ helix (yellow in Fig. 2A) between $\beta 5$ and $\beta 6$. The two $\beta$ sheets are orthogonal to each other and together form a $\beta$ barrel, which is a key structural feature of $\mathrm{PH}$ domains. One end of this $\beta$ barrel is blocked by two $\alpha$ helices (red in Fig. 2A) comprised of residues Glu461 to Leu496, while the other end of this $\beta$ barrel is a highly positively charged binding site for membrane lipid (Fig. 2D) according to several $\mathrm{PH} /$ lipid complex structures. We note that in our structure, the secondary structure region of each $\beta$ strand and $\alpha$ helix is quite different from the predicted results 

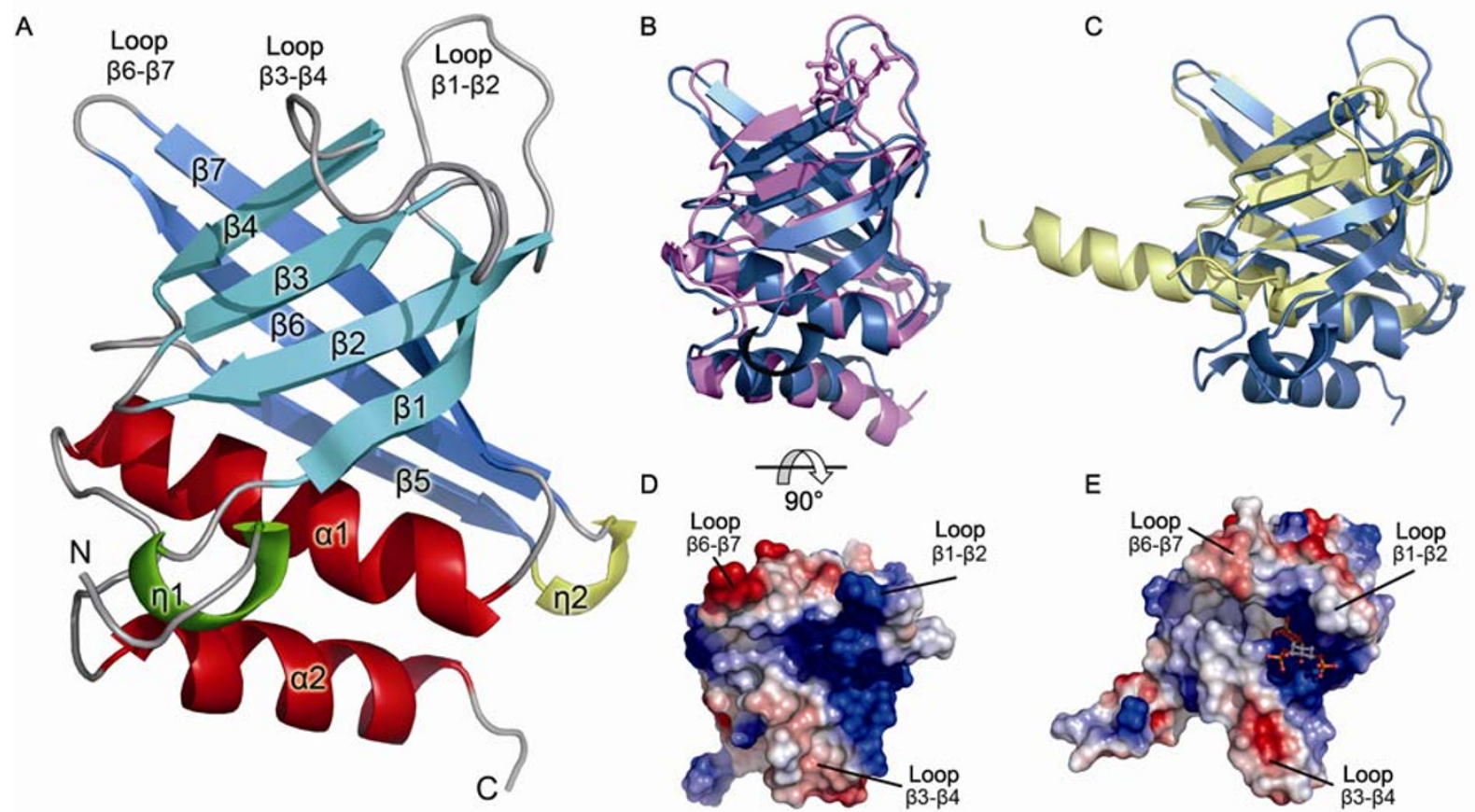

Figure 2. Overall structure of K2-PH. (A) Cartoon representation of K2-PH, with secondary structure elements and three important loops labeled appropriately. (B) Superposition of the crystal structure of K2-PH (blue) and the solution structure of K2-PH in complex with IP4 (PDB entry: 2LKO, magenta), with the IP4 molecule highlighted as ball-and-stick, shown in the same view as A. (C) Superposition of the crystal structure of K2-PH (blue) and the crystal structure of PKB-PH in free form (PDB entry: 1UNP, yellow), shown in the same view as A. (D) Electrostatic potential surface of K2-PH, calculated using PyMOL (DeLano Scientific, CA, USA), shown from the top of the lipid binding pocket. Red indicates areas with negative potential, while blue indicates positive potential. (E) Electrostatic potential surface of K2-PH bound to IP4 (PDB entry: 2LKO), with the IP4 molecule highlighted as ball-and-stick, shown in the same view as $D$.

in previous reports (Lee et al., 2011).

\section{The expansion of $\beta$ barrel caused by IP4 insertion}

The apo form of the K2-PH crystal structure allows us to compare it with the NMR structure of the same $\mathrm{PH}$ domain bound to IP4 (PDB entry: 2LKO). After superposition, the two structures of $\mathrm{K} 2-\mathrm{PH}$ shared an r.m.s.d. of $2.8 \AA$ for main-chain Ca atoms from Leu375 to Leu496, representing similar overall folds (Fig. 2B). Nevertheless, there is a significant electrostatic surface change in the lipid binding pocket between the apo and complex forms, which is apparently caused by the IP4 binding (Fig. 2D vs Fig. 2E). Also, upon the IP4 insertion, there is an expansion of the $\beta$ barrel from the side of the ligand binding pocket. The distance between the two anti-parallel $\beta$ sheets is $\sim 11 \AA$ in the free form crystal structure, versus $\sim 17 \AA$ in the complex structure (Fig. 3A). This difference is mostly caused by the movement of $\beta 3$ and $\beta 4$ strands, in which some residues directly interact with IP4, such as His419 (Fig. 3D). The movement seems to be con- sistent with the significant chemical shift changes of the $\beta 3$ and $\beta 4$ strands upon the IP4 binding to $\mathrm{K} 2-\mathrm{PH}$ as reported previously by Liu et al. (2011)

In order to further examine the expansion of the $\mathrm{PH} \beta$ barrel, the solution structure of K3-PH in free form (PDB entry: 2YS3, green in Fig. 3B) was also superimposed with our crystal structure of $\mathrm{K} 2-\mathrm{PH}$ in free form (blue in Fig. 3B). The entire $\beta$ barrels of two $\mathrm{PH}$ domains overlay well with each other, showing no obvious variance in the distance between two anti-parallel $\beta$ sheets. Therefore, we believe that the shrinking diameter of the $\beta$ barrel in our crystal structure is not a consequence of inter-molecular forces during crystal packing.

To our knowledge, such lipid-mediated $\beta$ barrel expansion has not been observed in other reported $\mathrm{PH}$ structures. For instance, the crystal structures of PKB-PH with or without lipid head group bound to it (Milburn et al., 2003) show nearly no difference of the $\beta$ barrel diameter (Fig. 3C). On the other hand, since those two structures are both crystal structures, there is a possibility that molecular packing in crystals limited the expansion ability of PKB-PH domain. 

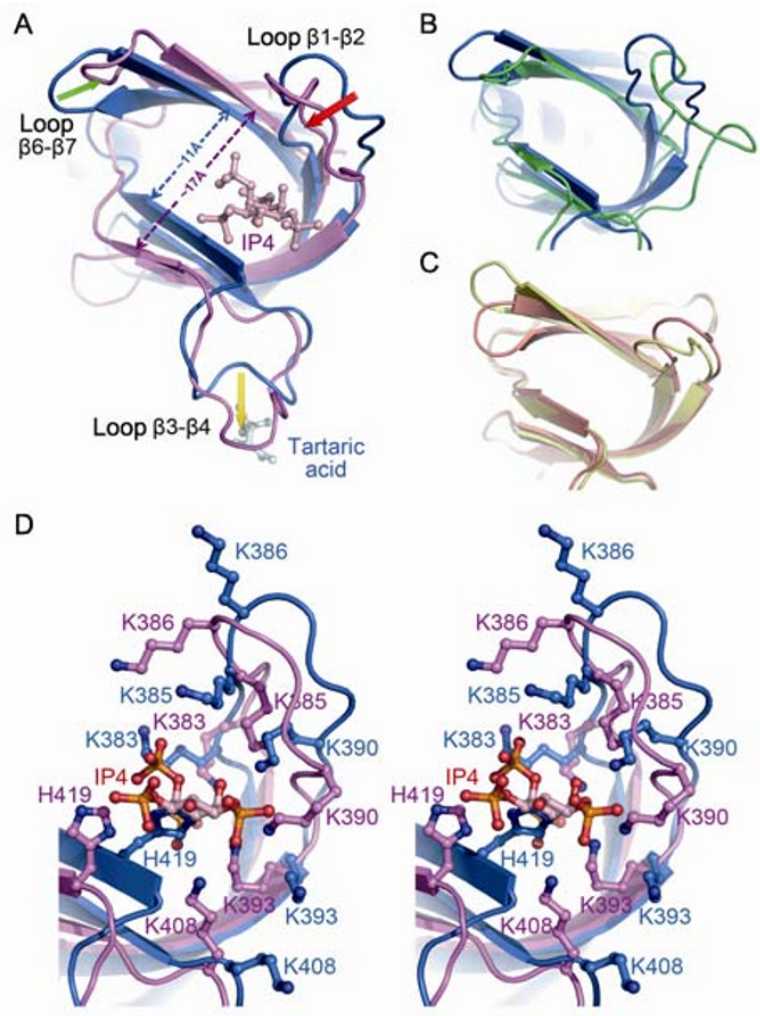

Figure 3. Conformational transition in K2-PH induced by IP4 binding. (A) Superposition of the crystal structure of $\mathrm{K} 2-\mathrm{PH}$ (blue) and the solution structure of $\mathrm{K} 2-\mathrm{PH}$ in complex with IP4 (PDB entry: 2LKO, magenta), shown from the top of the lipid binding pocket. Both the IP4 and the tartaric acid group near loop $\beta 3-\beta 4$ are highlighted as ball-and-stick, and colored similar to the protein they interact with. Three important loops surrounding the pocket, loop $\beta 1-\beta 2$, loop $\beta 3-\beta 4$ and loop $\beta 6-\beta 7$, are labeled; and their movements are indicated by arrows in different colors. (B) Superposition of the crystal structure of $\mathrm{K} 2-\mathrm{PH}$ in free form (b/ue) and the solution structure of K3-PH in free form (green), shown in the same view as A. (C) Superposition of the crystal structures of PKB-PH with (pink) or without IP4 binding (yellow), shown in the same view as A. (D) Stereo view of the positively-charged residues around the lipid binding pocket of K2-PH with (magenta) or without IP4 binding (blue). Residues Lys383, Lys385, Lys386, Lys390, Lys393, Lys408, His419, and small molecule IP4 are shown in ball-and-stick representation, while the main chains of $\mathrm{K} 2-\mathrm{PH}$ are shown in cartoon representation.

\section{The conformational transition of lipid binding pocket induced by IP4 binding}

Besides the $\beta$ barrel expansion, a couple of flexible loop regions of $\mathrm{K} 2-\mathrm{PH}$ also undergo conformational transitions that could be caused by IP4 interaction. Around the lipid binding pocket of $\mathrm{K} 2-\mathrm{PH}$, there are three loops, $\beta 1-\beta 2, \beta 3-\beta 4$, and $\beta 6-\beta 7$. A surface electrostatic analysis shows that the positively charged residues for IP4 binding are mainly on loop $\beta 1-\beta 2$ (Fig. 2D). Consistently, the superposition of two K2-PH structures reveals that, among those three loops, loop $\beta 1-\beta 2$ is the only one moving toward the pocket center due to the IP4 binding (red arrow in Fig. 3A). Also, the inward movement of loop $\beta 1-\beta 2$ can be readily observed in a comparison of the surface charges, where the free form $\mathrm{K} 2-\mathrm{PH}$ exposes more positively-charged protein surface (Fig. 2D) than the K2-PH in complex with IP4 (Fig. 2E). Consistently, the $\beta 1-\beta 2$ loop underwent dramatic chemical shift changes upon the IP4 binding (Liu et al., 2011).

For the other two loops when an IP4 binds to the pocket, loop $\beta 6-\beta 7$ shifts about $4 \AA$ (green arrow in Fig. $3 A$ ) following the expansion of strands $\beta 6$ and $\beta 7$, while loop $\beta 3-\beta 4$ adopts a distinct conformation (yellow arrow in Fig. $3 \mathrm{~A}$ ). These two loops are not directly involved in the interaction with IP4, but the chemical shifts also change in these regions, as consistent with their movement, which is probably due to the overall network rearrangement of the ligand binding pocket upon the IP4 binding. Note that the distinct conformation of the $\beta 3-\beta 4$ loop could also be partially induced by the tartaric acid group clamped between two K2-PH molecules during crystallization.

Consistent with the above-described lipid binding pocket change, the positively charged side chains around the lipid binding pocket of $\mathrm{K} 2-\mathrm{PH}$ reorient significantly. According to the K2-PH/IP4 complex structure and mutagenesis research, several Lys and His residues in the open pocket region are responsible for the interactions between K2-PH and IP4, including Lys383, Lys385, Lys386, Lys390, Lys393, Lys408, and His419 (Liu et al., 2011). The side chains of those residues orient toward the harbored IP4 to have electrostatic interactions with it (Fig. 3D). In the crystal structure of free form $\mathrm{K} 2-\mathrm{PH}$, the side chains of those lysine residues stay away from each other (Fig. 3D) and have higher $B$-factors than the side chains of the other residues in this region, indicating the remarkable flexibility of those side chains.

\section{CONCLUDING REMARKS}

Using x-ray crystallography, we have solved the crystal structure of human $\mathrm{K} 2-\mathrm{PH}$ domain to $1.9 \AA$, which represents the first crystal structure of the kindlin family. Comparison of our structure with the previously reported solution structure of $\mathrm{K} 2-\mathrm{PH}$ bound to IP4 revealed a significant "induced-fit" change. Notably, the core $\beta$ barrel of K2-PH expanded about $6 \AA$ due to the insertion of IP4; and the highly positively charged loop $\beta 1$ - $\beta 2$ (Phe382-Lys393) closes up over the IP4 to bind its negatively-charged phosphate groups. These conformational changes are remarkably consistent with their corresponding chemical shift changes as previously reported by Liu et al. (2011). Such allostery induced by IP4 binding is likely crucial for the tight binding of PIP3 to anchor kindlin-2 to the cytoplasmic membrane surface, thereby promoting the 
binding and activation of integrins.

\section{MATERIALS AND METHODS}

\section{Cloning, expression, and purification}

The nucleotide sequence encoding the human K2-PH domain (amino acids 328-499) was amplified and subcloned into the BamHI/Xhol sites of the pET-28a vector with an artificial SUMO-tag inside the Nhel/BamHI sites. The pET-28a-SUMO-K2-PH-transformed cells were induced with $0.2 \mathrm{mmol} / \mathrm{L} I P T G$, incubated overnight at $16^{\circ} \mathrm{C}$ and collected by centrifugation at $4000 \times g$ for $15 \mathrm{~min}$. The cell pellets were resuspended in lysis buffer $(20 \mathrm{mmol} / \mathrm{L}$ tris- $\mathrm{HCl} \mathrm{pH} \mathrm{8.0,150}$ $\mathrm{mmol} / \mathrm{L} \mathrm{NaCl}$ ) and lysed by sonication. The lysate was then pelleted by centrifugation at $16,000 \times g$ for $30 \mathrm{~min}$, after which the supernatant was loaded onto a gravity-flow column containing $5 \mathrm{~mL}$ Ni-NTA affinity resin (Qiagen, USA). After washing the column with ten column-volumes of lysis buffer, the His-SUMO-tag-fused K2-PH was eluted using elution buffer $(20 \mathrm{mmol} / \mathrm{L}$ tris- $\mathrm{HCl} \mathrm{pH} 8.0,150 \mathrm{mmol} / \mathrm{L}$ $\mathrm{NaCl}, 500 \mathrm{mmol} / \mathrm{L}$ imidazole). After cleavage of the His-SUMO-tag using Ulp1 enzyme at $4^{\circ} \mathrm{C}$ overnight, the proteins mixture was applied onto a Superdex-75 gel filtration column (GE Healthcare, USA) equilibrated with lysis buffer for further purification. Fractions containing $\mathrm{K} 2-\mathrm{PH}$ were collected and concentrated to about $50 \mathrm{mg} / \mathrm{mL}$ by ultrafiltration. The purified K2-PH with an artificial serine residue at its $\mathrm{N}$-terminus was used for further crystallographic studies.

\section{Crystallization}

The $\mathrm{K} 2-\mathrm{PH}$ was crystallized at $16^{\circ} \mathrm{C}$ using the hanging drop, vapor-diffusion method. The crystals were grown on a siliconized cover slip by equilibrating a mixture containing $1 \mu \mathrm{L}$ protein solution (50 mg/mL K2-PH in $20 \mathrm{mmol} / \mathrm{L}$ tris- $\mathrm{HCl} \mathrm{pH} 8.0,150 \mathrm{mmol} / \mathrm{L} \mathrm{NaCl}$ ) and $1 \mu \mathrm{L}$ reservoir solution $(0.1 \mathrm{~mol} / \mathrm{L}$ tris- $\mathrm{HCl} \mathrm{pH} 8.5,1.4 \mathrm{~mol} / \mathrm{L}$ ammonium tartrate dibasic) against $400 \mu \mathrm{L}$ reservoir solution. Single crystals formed after 2 months with a largest dimension of about $0.1 \mathrm{~mm}$. For cryogenic experiment, a suitable cryoprotectant was prepared as reservoir solution plus $15 \%(v / v)$ glycerol for crystal soaking; and the cryoprotected K2-PH crystals were flash-frozen by liquid nitrogen for future data collection.

\section{Data collection, phasing, and model refinement}

On the in-house x-ray source, MicroMax 007 generator (Rigaku, Japan) combining Varimax HR optics (Rigaku, Japan), of the Institute of Biophysics, Chinese Academy of Sciences, K2-PH crystals at $100 \mathrm{~K}$ diffracted to $1.9 \AA$ resolution at a wavelength of $1.5418 \AA$. A native set of x-ray diffraction data was collected with Satun 944+ CCD detector (Rigaku, Japan) with an exposure time of $15 \mathrm{~s}$ per image, and was indexed and processed using HKL2000 (Otwinowski and Minor, 1997). The space group of collected dataset is $\mathrm{P} 4_{1} 2_{1} 2$ or $\mathrm{P}_{3} 2_{1} 2$, which could not be determined until solving the phase problem. Data-collection statistics are given in Table 1.

Since the solution structure of K2-PH has been reported (Liu et al.,
2011), molecular replacement was performed with PHENIX.phaser (McCoy et al., 2007) to solve the phasing problem. Both the solution structures of K2-PH (PDB entry: 2LKO) and K3-PH (PDB entry: 2YS3) were employed as the starting model for molecular replacement; and the latter one gave out reasonable phase solution in the space group of $\mathrm{P}_{3} 2_{1} 2$. The final model was manually adjusted in COOT (Emsley and Cowtan, 2004) and refined with PHENIX.refine (Adams et al., 2010). Refinement statistics are given in Table 1.

\section{Coordinates deposition}

Coordinates of the refined model of K2-PH domain and its experimental structural factors have been deposited to Protein Data Bank (http://www.pdb.org/) with the accession number 4F7H.

\section{ACKNOWLEDGEMENTS}

We thank Prof. Jun Qin (Cleveland Clinic, $\mathrm{OH}, \mathrm{USA}$ ) for his valuable comments and critical discussion. We also thank Ms. Ping Shan in our laboratory for her kindly help during this study. We are very grateful to the staff of the Structural Biology Core Facility in the Institute of Biophysics, Chinese Academy of Sciences, for their technical assistance, especially to Ms. Ya Wang and Mr. Yi Han. This work was supported by the 973 Program of the Ministry of Science and Technology of China (Grant Nos. 2011CB911101 and 2012CB910304).

\section{ABBREVIATIONS}

FERM, four-point-one, ezrin, radixin, moesin; NMR, nuclear magnetic resonance; $\mathrm{PH}$ domain, pleckstrin homology domain

\section{REFERENCES}

Adams, P.D., Afonine, P.V., Bunkóczi, G., Chen, V.B., Davis, I.W., Echols, N., Headd, J.J., Hung, L.W., Kapral, G.J., Grosse-Kunstleve, R.W., et al. (2010). PHENIX: a comprehensive Python-based system for macromolecular structure solution. Acta Crystallogr D Biol Crystallogr 66, 213-221.

Anthis, N.J., Wegener, K.L., Ye, F., Kim, C., Goult, B.T., Lowe, E.D., Vakonakis, I., Bate, N., Critchley, D.R., Ginsberg, M.H., et al. (2009). The structure of an integrin/talin complex reveals the basis of inside-out signal transduction. EMBO J 28, 3623-3632.

Emsley, P., and Cowtan, K. (2004). Coot: model-building tools for molecular graphics. Acta Crystallogr D Biol Crystallogr 60, 2126-2132.

García-Alvarez, B., de Pereda, J.M., Calderwood, D.A., Ulmer, T.S., Critchley, D., Campbell, I.D., Ginsberg, M.H., and Liddington, R.C. (2003). Structural determinants of integrin recognition by talin. Mol Cell 11, 49-58.

Harburger, D.S., Bouaouina, M., and Calderwood, D.A. (2009). Kindlin-1 and -2 directly bind the C-terminal region of beta integrin cytoplasmic tails and exert integrin-specific activation effects. J Biol Chem 284, 11485-11497. 
Hynes, R.O. (2002). Integrins: bidirectional, allosteric signaling machines. Cell 110, 673-687.

Kim, M., Carman, C.V., and Springer, T.A. (2003). Bidirectional transmembrane signaling by cytoplasmic domain separation in integrins. Science 301, 1720-1725.

Lee, J.H., An, J.Y., Park, H., Kim, H.J., and Eom, S.H. (2011). Crystallization and preliminary $\mathrm{x}$-ray crystallographic analysis of the human kindlin-2 PH domain. Acta Crystallogr Sect $F$ Struct Biol Cryst Commun 67, 696-699.

Lemmon, M.A. (2008). Membrane recognition by phospholipid-binding domains. Nat Rev Mol Cell Biol 9, 99-111.

Liu, J., Fukuda, K., Xu, Z., Ma, Y.Q., Hirbawi, J., Mao, X., Wu, C., Plow, E.F., and Qin, J. (2011). Structural basis of phosphoinositide binding to kindlin-2 protein pleckstrin homology domain in regulating integrin activation. J Biol Chem 286, 43334-43342.

Luo, B.H., Carman, C.V., and Springer, T.A. (2007). Structural basis of integrin regulation and signaling. Annu Rev Immunol 25, 619-647.

Ma, Y.Q., Qin, J., Wu, C., and Plow, E.F. (2008). Kindlin-2 (Mig-2): a co-activator of beta3 integrins. J Cell Biol 181, 439-446.

McCoy, A.J., Grosse-Kunstleve, R.W., Adams, P.D., Winn, M.D., Storoni, L.C., and Read, R.J. (2007). Phaser crystallographic software. J Appl Crystallogr 40, 658-674.

Milburn, C.C., Deak, M., Kelly, S.M., Price, N.C., Alessi, D.R., and van Aalten, D.M. (2003). Binding of phosphatidylinositol 3,4,5-trisphosphate to the pleckstrin homology domain of protein kinase B induces a conformational change. Biochem J 375, 531-538.

Montanez, E., Ussar, S., Schifferer, M., Bösl, M., Zent, R., Moser, M., and Fässler, R. (2008). Kindlin-2 controls bidirectional signaling of integrins. Genes Dev 22, 1325-1330.

Moser, M., Legate, K.R., Zent, R., and Fässler, R. (2009). The tail of integrins, talin, and kindlins. Science 324, 895-899.

Moser, M., Nieswandt, B., Ussar, S., Pozgajova, M., and Fässler, R. (2008). Kindlin-3 is essential for integrin activation and platelet aggregation. Nat Med 14, 325-330.

Otwinowski, Z., and Minor, W. (1997). Processing of x-ray diffraction data collected in oscillation mode. Methods Enzymol 276, 307-326.

Perera, H.D., Ma, Y.Q., Yang, J., Hirbawi, J., Plow, E.F., and Qin, J. (2011). Membrane binding of the $\mathrm{N}$-terminal ubiquitin-like domain of kindlin-2 is crucial for its regulation of integrin activation. Structure 19, 1664-1671.

Plow, E.F., Qin, J., and Byzova, T. (2009). Kindling the flame of integrin activation and function with kindlins. Curr Opin Hematol 16, 323-328.

Qin, J., Vinogradova, O., and Plow, E.F. (2004). Integrin bidirectional signaling: a molecular view. PLoS Biol 2, e169.

Qu, H., Tu, Y., Shi, X., Larjava, H., Saleem, M.A., Shattil, S.J., Fukuda, K., Qin, J., Kretzler, M., and Wu, C. (2011). Kindlin-2 regulates podocyte adhesion and fibronectin matrix deposition through interactions with phosphoinositides and integrins. J Cell Sci 124, 879-891.

Rogalski, T.M., Mullen, G.P., Gilbert, M.M., Williams, B.D., and Moerman, D.G. (2000). The UNC-112 gene in Caenorhabditis elegans encodes a novel component of cell-matrix adhesion structures required for integrin localization in the muscle cell membrane. J Cell Biol 150, 253-264.

Shi, X., Ma, Y.Q., Tu, Y., Chen, K., Wu, S., Fukuda, K., Qin, J., Plow, E.F., and Wu, C. (2007). The MIG-2/integrin interaction strengthens cell-matrix adhesion and modulates cell motility. J Biol Chem 282, 20455-20466.

Siegel, D.H., Ashton, G.H., Penagos, H.G., Lee, J.V., Feiler, H.S., Wilhelmsen, K.C., South, A.P., Smith, F.J., Prescott, A.R., Wessagowit, V., et al. (2003). Loss of kindlin-1, a human homolog of the Caenorhabditis elegans actin-extracellular-matrix linker protein UNC-112, causes Kindler syndrome. Am J Hum Genet 73, 174-187.

Vinogradova, O., Velyvis, A., Velyviene, A., Hu, B., Haas, T., Plow, E., and Qin, J. (2002). A structural mechanism of integrin alpha(Illb)beta(3) "inside-out" activation as regulated by its cytoplasmic face. Cell 110, 587-597.

Zhang, Y., and Wang, H. (2012). Integrin signalling and function in immune cells. Immunology 135, 268-275. 